\title{
Equipartitioning and Balancing Points of Polygons
}

\author{
Shunmugam Pillay \& Poobhalan Pillay \\ School of Mathematical Sciences \\ University of KwaZulu-Natal \\ pillaysc@ukzn.ac.za \& pillayph@ukzn.ac.za
}

\begin{abstract}
The centre of mass $G$ of a triangle has the property that the rays to the vertices from $G$ sweep out triangles having equal areas. We show that such points, termed equipartitioning points in this paper, need not exist in other polygons. A necessary and sufficient condition for a quadrilateral to have an equipartitioning point is that one of its diagonals bisects the other. The general theorem, namely, necessary and sufficient conditions for equipartitioning points for arbitrary polygons to exist, is also stated and proved. When this happens, they are in general, distinct from the centre of mass. In parallelograms, and only in them, do the two points coincide.
\end{abstract}

This paper provides a specific scenario that can be utilised to stimulate learners into investigating a geometrical problem through the questioning process. The investigation leads to conjectures that are verifiable with the aid of computer software like the Geometer's Sketchpad, and then the formal proofs verifying the conjectures are made.

Traditionally the function of proof has been seen to be to verify or justify a mathematical statement. It is well known that proofs, especially in geometry, are a major problem for learners. For most learners mathematical statements (theorems) seem obvious and they see no need for proofs. Often, they do not appreciate a need for proofs and get frustrated when asked to prove a given theorem or "to prove" a statement from a textbook or a teacher (De Villiers, 2003, p. 5).

To stimulate genuine interest in proof, De Villiers (1997), Chazan (1990) and others have argued that it requires that students are inducted early into the art of problem posing, allowing sufficient opportunity for exploration, conjecturing, refuting, reformulating and explaining. Movshovitz-Hadar (1988) has similarly suggested stimulating presentations of results that solicit the surprise and curiosity of students so that they are susceptible to responsive proofs, which leave them with "an appreciation of the invention, along with a feeling of becoming wiser" (p. 15).

Pólya emphasizes that the students' interest and motivation must stem from mathematics itself. It is the duty of the teacher to select tasks for the students that are: not too difficult and not too easy, natural and interesting and appropriate to their knowledge. Mathematics should also be used to develop problem solving skills and gain confidence and hence students "may experience the tension and enjoy the triumph of discovery" (1945, p. v).

Further the reform movement in mathematics education requires the teacher to provide tasks that are stimulating, relevant and foster growth in knowledge. This can be achieved by helping students connect mathematical ideas and concepts to real life situations (National Council of Teachers of Mathematics, 1991).

To this end, the problem in Figure 1 can be posed and investigated in a Grade 11 class.

Such an investigation may proceed along the following lines. 
Mr Baker makes biscuits that are in the shape of polygons. He has templates of triangles, quadrilaterals, pentagons and so on, but although all of them are convex, most of them have random dimensions.

He finds a point in his triangle template that has the property that the three triangles formed by joining the point to the three vertices all have the same area. This pleases him, for he can then sell each piece separately at the same price.

He calls such points, if they can be found, equipartitioning points.

Investigate the following.

1. How does he locate the equipartitioning point inside the triangle and how can he be certain that the point is the correct one? How many such points are there?

2. Can he do the same with a quadrilateral? That is, can all quadrilaterals be divided into four equal triangles in the manner described?

3. Justify your answer. If it cannot be done, for which quadrilaterals would $\mathrm{Mr}$ Baker be able to locate at least one equipartitioning point?

4. Investigate the same question for convex polygons having five or more sides.

5. Mr Baker is aware that all polygons have balancing points, also called centres of mass, namely, points at which the mass of the polygon may be presumed to be concentrated. (Here he regards the polygon as a plate having uniform density). Would the balancing point of a polygon be the point Mr Baker is looking for?

Figure 1: The problem

\section{The investigation}

\section{The square, rectangle and parallelogram}

Learners should have no difficulty in identifying the equipartitioning points in these shapes as the points of intersection of the diagonals. Finally they should explain why such points work.

\section{The triangle}

1. Using appropriate software, locate such points for a predetermined number of triangles. Also ascertain how many such points there are.

2. Use the points obtained to conjecture a rule by which these points may be located without using technology.

3. Give a formal proof of your assertions in 1 and 2.

We believe that with a little help maybe, all learners would come to the conclusion that every triangle has exactly one equipartitioning point, and that it is situated at the point of intersection of (any) two of its medians. The uniqueness of the equipartitioning point also can be used to argue that for any triangle all three medians go through the same point, namely, it's equipartitioning point. This leads to a secondary conjecture.

\section{The kite}

Use appropriate software again to determine whether kites have equipartitioning points. Make a conjecture and give a formal proof of your claim.

\section{The general quadrilateral}

Do the same for trapeziums and cyclic quadrilaterals. What do you conclude? Which quadrilaterals have equipartitioning points? Are these the only ones? 


\section{Centres of mass}

Each learner could then be asked to plot the equipartitioning point of stiff triangular boards, and then try to support the triangle with a finger held up vertically under the board, at the point where the equipartitioning is located. If the construction is accurate, the board will balance perfectly. It is known that all polygons possess such balancing points. The terms centres of mass, centre of gravity and centroid are also used. Hence, for triangles, the equipartitioning point coincides with the centre of mass.

\section{Formal statements and proofs}

Formal definition

Let $A_{1} A_{2} \ldots A_{n}$ be a polygon. An interior point $Q$ of the polygon is called an equipartitioning point of $A_{1} A_{2} \ldots A_{n}$ if the $n$ triangles $Q A_{1} A_{2}, Q A_{2} A_{3}, \ldots, Q A_{n-1} A_{n}, Q A_{n} A_{1}$ all have the same area.

In Figure 2 we have that $\frac{\text { area } A B D}{\text { area } A D C}=\frac{B D}{D C}$. Hence if $A D$ is a median, $B D=D C$, so area $A B D=$ area $A D C$.

That is, a median bisects the area of a triangle.

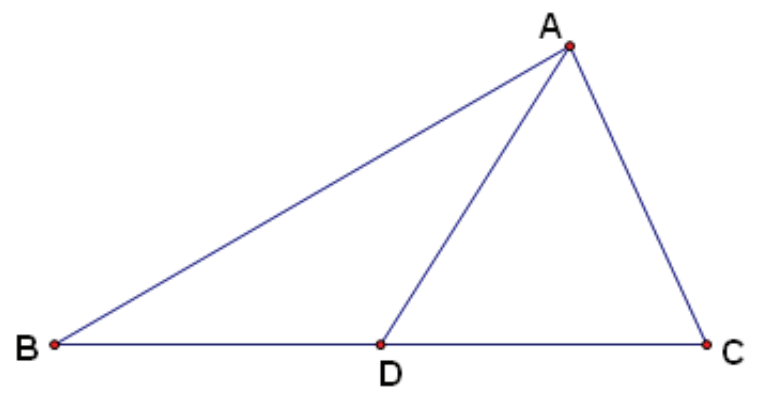

Figure 2

\section{Theorem 1}

A triangle has a unique equipartitioning point, namely, the point of concurrency of its medians.

\section{Proof}

We first prove that the point $G$ of concurrency of the medians of a triangle is an equipartitioning point.

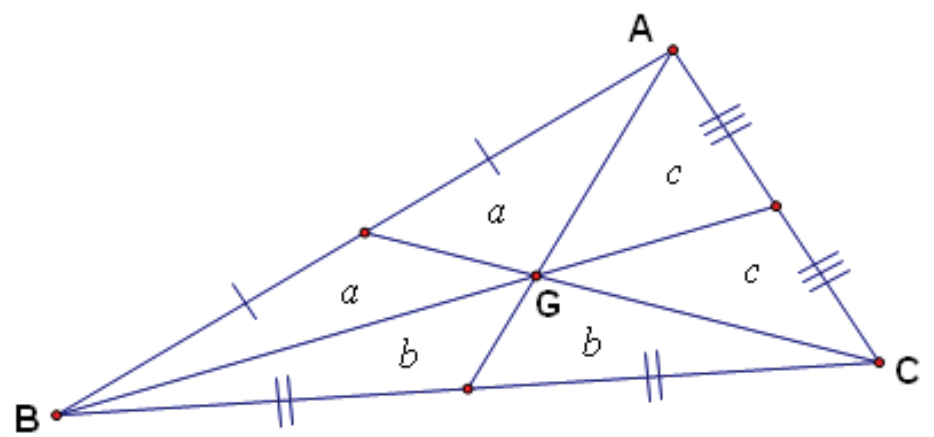

Figure 3

The symbols $a, b, c$ in Figure 3 refer to the areas of the triangles. Now $2 a+b=2 c+b$ and $a+2 b=a+2 c$ lead to $2 a=2 b=2 c$. So $G$ is an equipartitioning point. 
Conversely, let $Q$ be any equipartitioning point of $\triangle A B C$ Produce $B Q$ to meet $A C$ at $D$. Let $a, x$ and $y$ be the areas as indicated in Figure 4. Since $Q$ is an equipartitioning point, $a=x+y$.

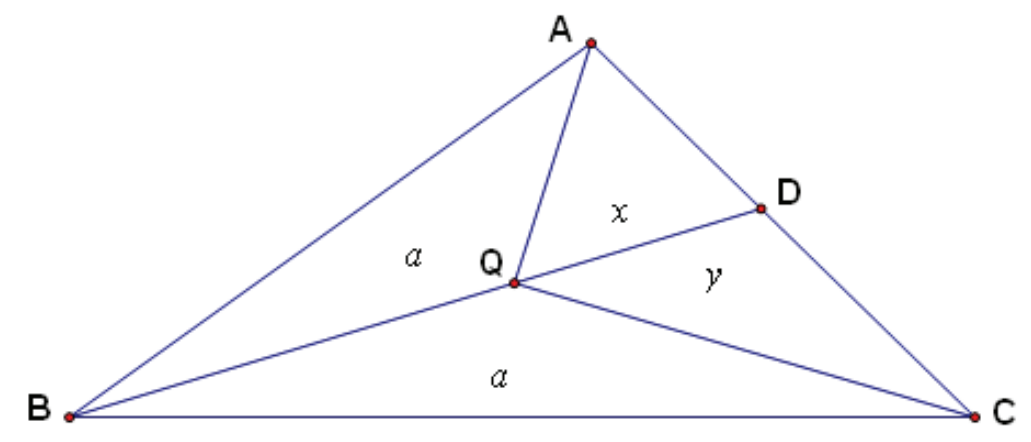

Figure 4

Now $\frac{B Q}{Q D}=\frac{a}{x}=\frac{a}{y}$ so $x=y$, hence $A D=D C$, proving that $Q$ lies on the median through $B$. By symmetry, $Q$ also lies on the median through $A$. Hence, $Q$ is the point of concurrency $G$ of the medians. We have shown that $\mathrm{G}$ is the only equipartitioning point.

\section{The case for a quadrilateral}

The following is of independent interest.

\section{Theorem 2}

A diagonal of a quadrilateral (not necessarily convex) bisects the area of a quadrilateral $\Leftrightarrow$ the diagonal bisects the other diagonal (see Coxeter \& Greitzer, 1967, pp. 54-55).

\section{Proof}

Suppose one of the diagonals bisects the other.

Refer to Figure 5. Let the area of $\triangle A D E=a$ and the area of $\triangle A B E=b$.

Then it is an easy matter to see that the same diagonal bisects the area as well.
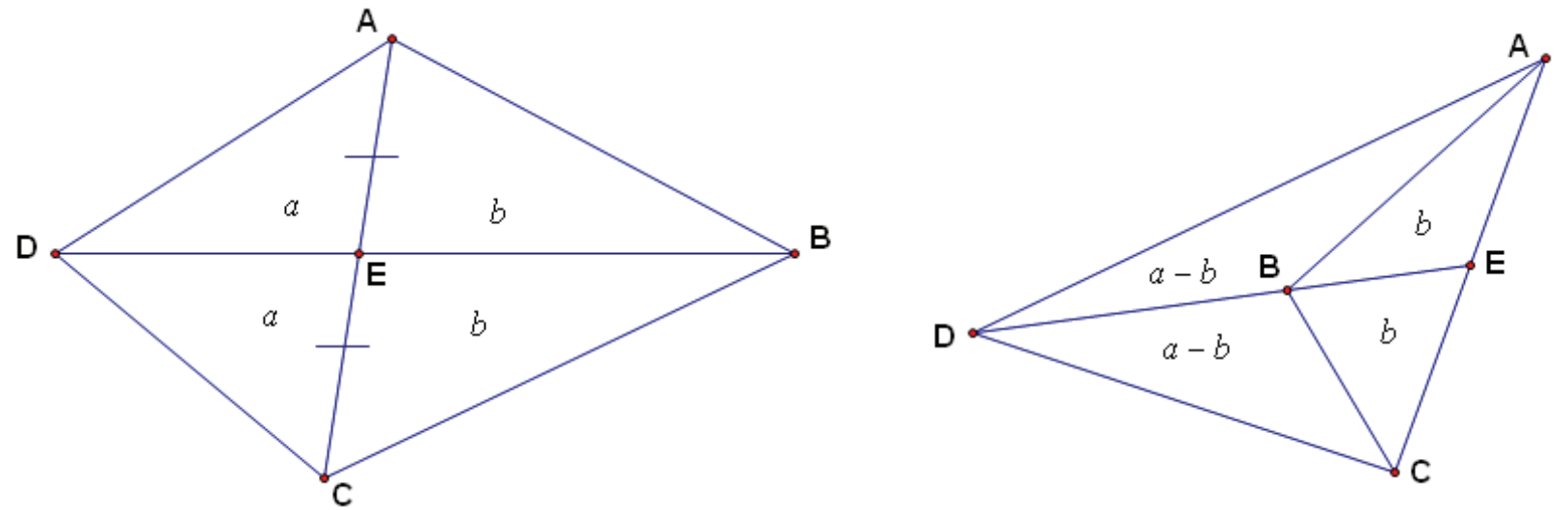

Figure 5

Conversely, let $A B C D$ be a quadrilateral, not necessarily convex, such that $D B$ bisects the area of $A B C D$. Draw the altitudes $A E$ and $C F$ of $\triangle A B D$ and $\triangle D B C$ as shown in Figure 6.

Since the area of $\triangle A D B$ is equal to the area of $\triangle C D B$, we have $\frac{1}{2} D B \cdot A E=\frac{1}{2} D B . C F$, so $A E=C F$.

Let us first assume that $E$ and $F$ are distinct points. Since $A E / / F C, A E C F$ is a parallelogram. Hence the midpoint of diagonal $A C$ is on $F E$ (and hence on $D B$ or $D B$ produced), proving that one of the diagonals bisects the other. 
If $E=F, A E C$ is a straight line, $D B$ bisects $A C$ and is perpendicular to it. (In this case $A B C D$ is a kite). This completes the proof.
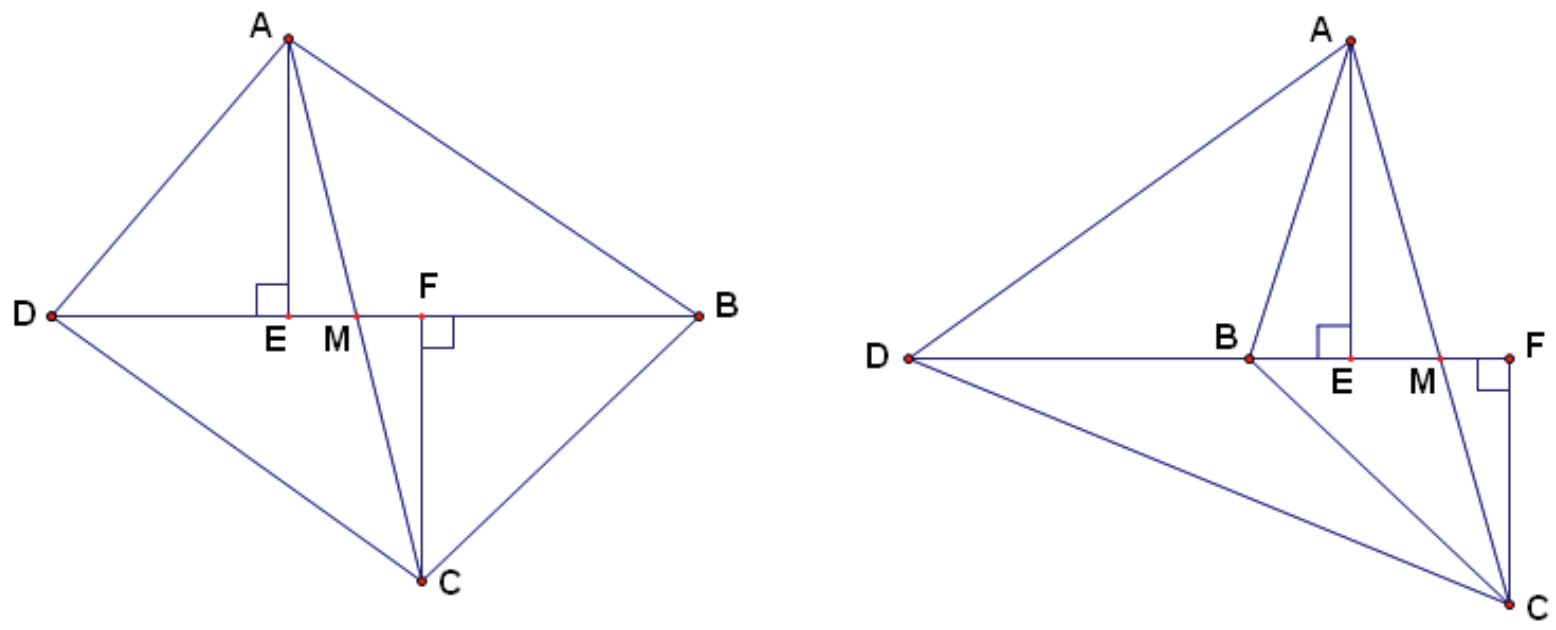

Figure 6

Whereas all polygons have centres of gravity, not all polygons have equipartitioning points, as the case for triangles seems to suggest. The next result provides a necessary and sufficient condition for a quadrilateral to have an equipartitioning point. The convex and non-convex cases are treated separately.

\section{Theorem 3}

A quadrilateral has an equipartitioning point $Q \Leftrightarrow$ one of its diagonals bisects the other, and then $Q$ is the midpoint of the first diagonal (see Gilbert, Krusemeyer, \& Larson, 1993).

\section{Proof}

We consider two cases, one where the quadrilateral is convex, and the other where it is not convex.

Case 1

Let $A B C D$ be a convex quadrilateral such that $A C$ bisects $B D$ (see Figure 7).

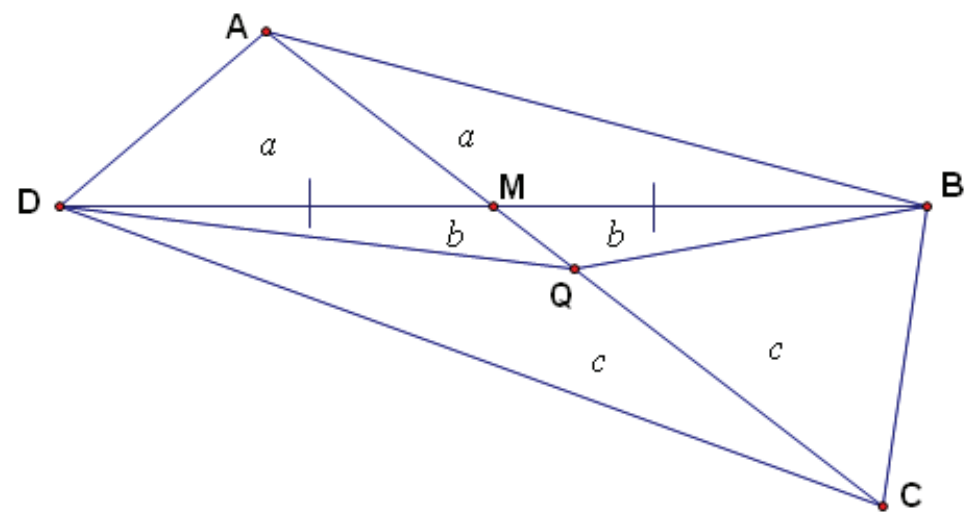

Figure 7

Let $Q$ be the midpoint of $A C$ and let $a, b$ and $c$ be the indicated areas. Then $c=a+b$, proving that the midpoint $Q$ of diagonal $A C$ is an equipartitioning point.

Conversely, suppose a convex quadrilateral $A B C D$ has an equipartitioning point $Q$ (see Figure 8).

Then diagonals $Q B$ and $Q D$ bisect the areas of quadrilaterals $A B C Q$ and $A Q C D$ respectively. Hence from Theorem 1, both $Q B$ and $D Q$ contain the midpoint $M$ of $A C$. 


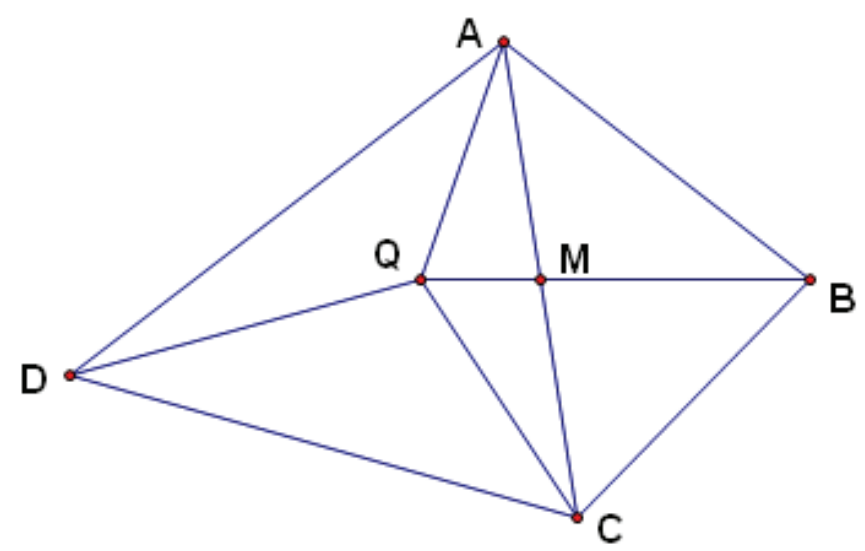

Figure 8

Suppose that $Q$ is different from $M$. The straight line through $Q$ and $M$ contains $D$ and $B$, hence $D Q B$ is the diagonal $D B$ and it bisects $A C$. But triangles $A D Q$ and $A B Q$ have the same area, so $D Q=Q B$. We have shown that in the case where $Q$ and $M$ are distinct, diagonal $D B$ bisects diagonal $A C$ and the equipartitioning point $Q$ is the midpoint of $D B$.

Now suppose the equipartitioning point $Q=M$, the midpoint of $A C$. The diagonal $A C$ then bisects the area of $A B C D$. From Theorem 2, diagonal $A C$ bisects diagonal $D B$ and $Q(=M)$ is the midpoint of $A C$. So the theorem is proved for convex quadrilaterals.

\section{Case 2}

Let $A B C D$ be a quadrilateral with $\angle B C D>180^{\circ}$ (see Figure 9). We shall prove that $A B C D$ has an equipartitioning point $Q \Leftrightarrow A C$ (produced) bisects $D B$, and then $Q$ is the midpoint of $A C$.

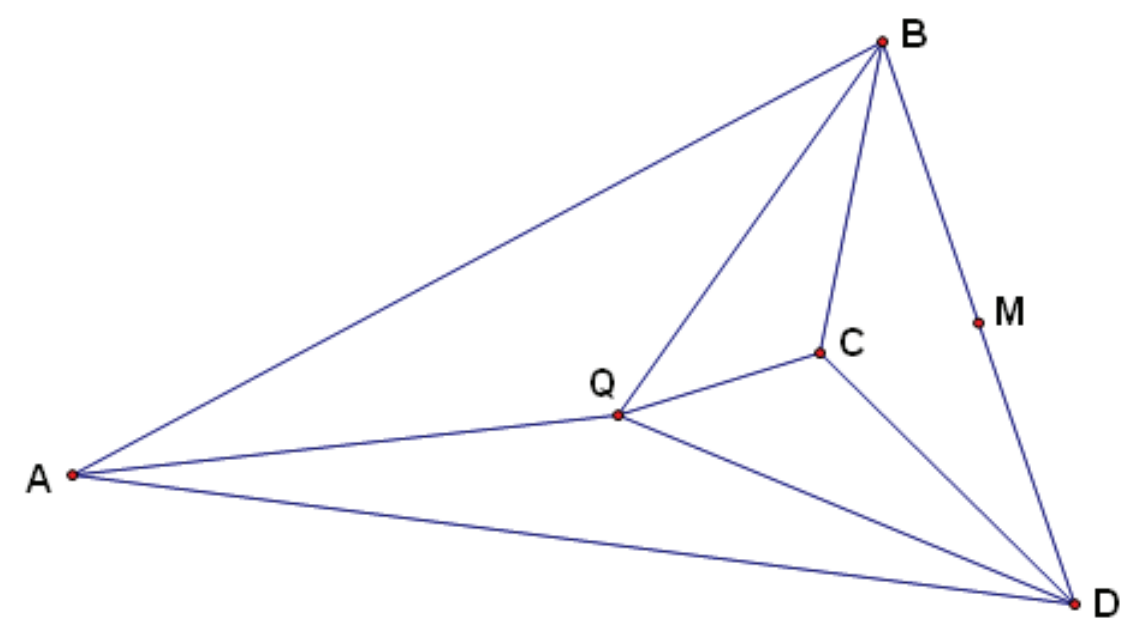

Figure 9

One direction is straightforward; if $A C$ produced bisects the diagonal $B D$, then it is easy to see that if $Q$ is the midpoint of $A C$, then the areas of triangles $Q A B, Q B C, Q C D$, and $Q D A$ are all equal.

Suppose then $A B C D$ has an equipartitioning point $Q$ in its interior. The areas of triangles $Q A B, Q B C$, $Q C D$ and $Q D A$ are all equal. Then diagonals $A Q$ and $Q C$ bisect the areas of (convex) quadrilaterals $A B Q D$ and $B Q D C$ respectively. From Theorem 2, the midpoint $M$ of $B D$ lies on $A Q$ and $Q C$ produced. Since $M$ is in the exterior of $A B C D, A, Q, C$ and $M$ are collinear. Then $B Q$ bisects the area $\triangle A B C$ so $Q$ is the midpoint of $A C$. Also $A C$ bisects $B D$, so we are done. 


\section{Remarks}

Learners may now be asked:

1. Explain why parallelograms and kites always have equipartitioning points.

2. Prove that a trapezium has an equipartitioning point if, and only if, it is a parallelogram.

\section{The general case}

We are now in a position to generalise to an arbitrary polygon.

\section{Theorem 4}

Let $A_{1} A_{2} \ldots A_{n}$ be a polygon. For any three adjacent vertices $X, Y, Z$ of the polygon, let $Y^{\prime}$ be the midpoint of $X Z$. Then $A_{1} A_{2} \ldots A_{n}$ has an equipartitioning point $Q \Leftrightarrow$ the $n$ lines $P P^{\prime}$, as $P$ runs through the $n$ vertices, are concurrent, and then the point of concurrency is the equipartitioning point of $A_{1} A_{2} \ldots A_{n}$.

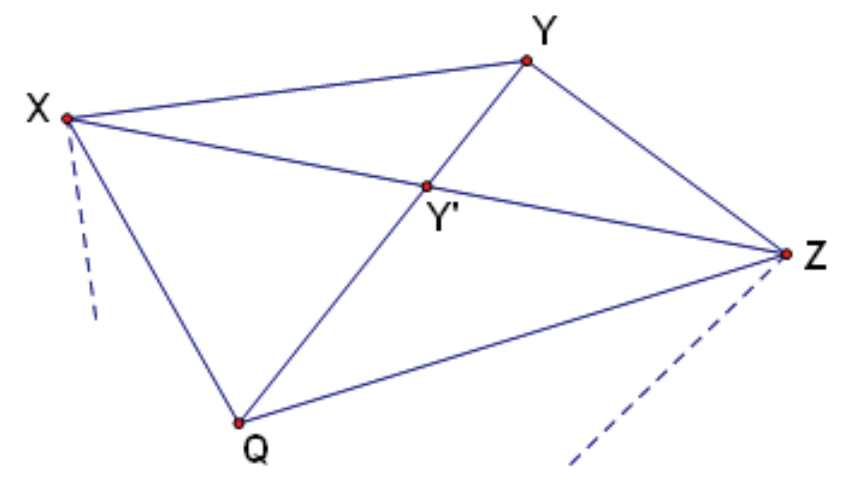

Figure 10

\section{Proof}

Suppose the polygon (refer to Figure 10) has an equipartitioning point $Q$. Let $X, Y, Z$ be adjacent vertices. From Theorem $2 Q$ lies on $Y Y^{\prime}$ (possibly produced). It follows that $Q$ lies on each of the $n$ lines $P P^{\prime}$, as $P$ runs through the $n$ vertices, so the $n$ lines are concurrent. Conversely, if $Q$ lies on each such $Y Y^{\prime}$ (possibly produced), it is easy to see that for any three adjacent vertices $X, Y, Z$, triangles $Q X Y$ and $Q Y Z$ have equal area, so $Q$ is an equipartitioning point, proving the theorem.

\section{Remarks}

Learners may be asked to verify the following:

1. In the special case where the polygon is a triangle, the three lines mentioned above are just the medians, and, since the medians of a triangle are always concurrent, all triangles have equipartitioning points.

2. In the case of a quadrilateral the concurrency of the four lines above is equivalent to the statement that one of the diagonals bisects the other, and then the point of concurrency is the midpoint of the bisecting diagonal.

\section{Balancing points}

In contrast to equipartitioning points, balancing points (also called centres of mass, centres of gravity or centroids) of all plane regions, and in particular, of all polygons, always exist. The following result (Wales, 2010) provides a method to locate balancing points of polygonal plane regions. It should be mentioned that the centre of mass or centroid of equal point masses placed at the vertices of a polygon, in general, do not coincide with the centre of mass of a polygonal plane region. 


\section{Theorem 5}

Let $R$ be a plane region bounded by a polygon (see Figure 11). Suppose $R$ is subdivided into two regions $R_{1}$ and $R_{2}$ having areas $A_{1}$ and $A_{2}$ respectively. Let $G_{1}$ and $G_{2}$ be the centres of mass of $R_{1}$ and $R_{2}$. Then the centre of mass $G$ of $R$ is a point in the interior of the line segment $G_{1} G_{2}$ satisfying the condition $A_{1} \cdot G_{1} G=A_{2} \cdot G_{2} G$.

In particular, if the two areas are equal, then $G$ is the midpoint of $G_{1} G_{2}$.

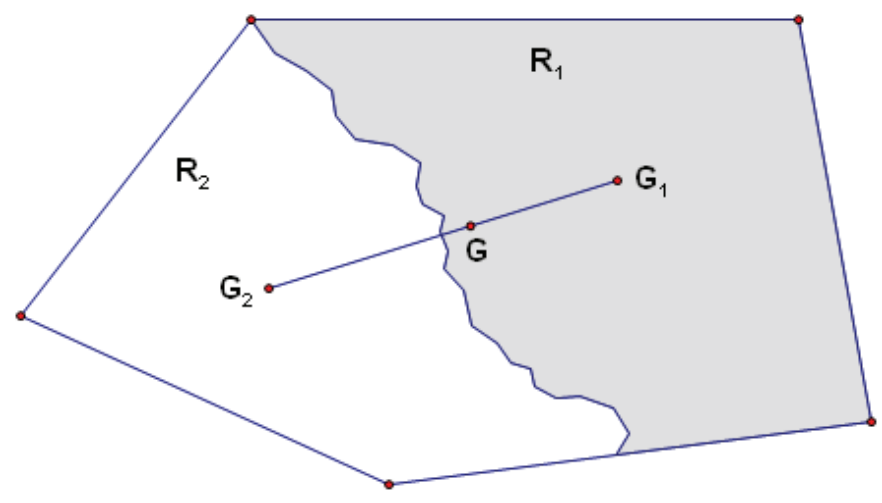

Figure 11

The next theorem classifies those plane quadrilaterals having equipartitioning points coinciding with their centre of mass.

\section{Theorem 6}

Let $A B C D$ be a quadrilateral that has an equipartitioning point $Q$. Then $Q$ is the centre of mass of $A B C D$ if, and only if, $A B C D$ is a parallelogram.

\section{Proof}

Let $A B C D$ be a parallelogram whose diagonals $A C$ and $B D$ intersect at $M$.
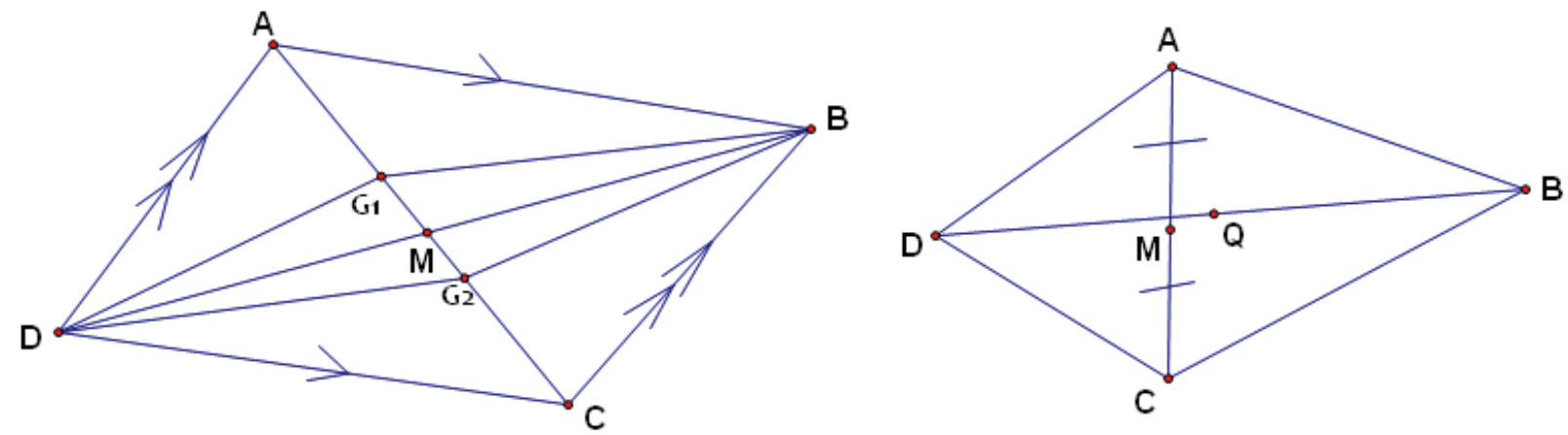

Figure 12

The centre of mass $G_{1}$ of $\triangle A B D$ lies on $A M$ with $G_{1} M=\frac{1}{3} A M$. The centre of mass $G_{2}$ of $\triangle C B D$ lies on $C M$ with $G_{2} M=\frac{1}{3} C M$. Since $A M=C M, M$ is the midpoint of $G_{1} G_{2}$. Since $\triangle A B D$ and $\triangle C B D$ have equal areas, $G$, the centre of mass of $A B C D$, is the midpoint of $G_{1} G_{2}$, by Theorem 5. That is, $G=M$. Since the diagonals partition a parallelogram into four equal areas, $M=Q$, the equipartitioning point. So $Q=G$ is the centre of mass of $A B C D$.

Conversely, let quadrilateral $A B C D$ have equipartitioning point $Q$, and further, suppose $Q=G$, the centre of mass of $A B C D$.

Let the diagonals intersect at $M$. By Theorem 4, we may assume $A M=C M$ and $B Q=D Q$. Suppose $A Q C$ is not a straight line. Let $G_{1}$ and $G_{2}$ be the centres of mass $\triangle C B D$ and $\triangle A B D$ respectively. Then $G_{1}$ lies on $C Q$ and $G_{2}$ lies on $A Q$ and $G_{1} Q G_{2}$ is a triangle. But $G_{1} G G_{2}$ is a straight line, contradicting $Q=G$. Hence $A Q C$ is a straight line. It follows that $A C$ and $B D$ bisect each other. Hence $A B C D$ is a parallelogram. 


\section{The parallelogram technique}

The following construction provides a simple technique to find the centre of mass of an arbitrary plane quadrilateral.

Trisect the four sides and complete the parallelogram determined by these eight points (see Figure 13). The diagonals of the parallelogram meet at the centre of mass of the plane quadrilateral.

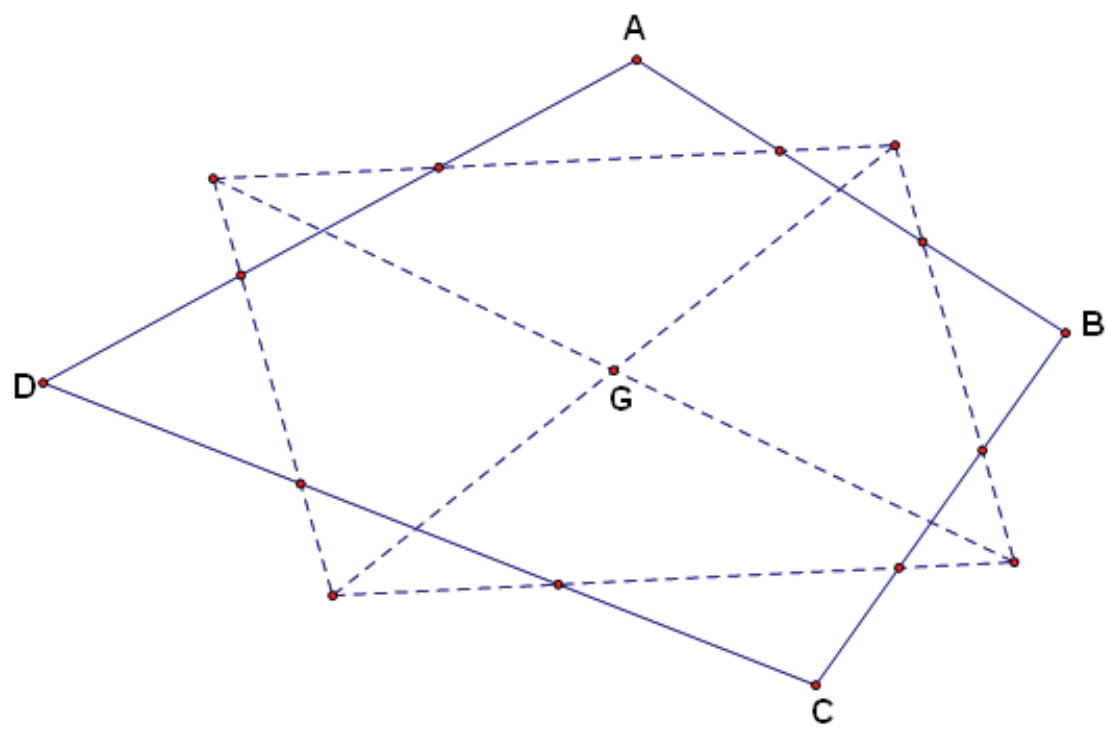

Figure 13

\section{Discussion}

We trust that this paper will encourage teachers and learners to investigate different partitionings of polygons into parts of equal area, because it is a very rich topic with some challenging, still unsolved problems (see for example Croft, Falconer, \& Guy, 1991).

\section{References}

Chazan, D. (1990). Quasi-empirical views of mathematics and mathematics teaching. Interchange, 21(1), 14-23. doi: 10.1007/BF01809606

Coxeter, H. S. M., \& Greitzer, S. L. (1967). Geometry revisited. Washington, DC: Mathematical Association of America.

Croft, H. T., Falconer, K. J., \& Guy, R. K. (1991). Unsolved problems in geometry. NewYork: SpringerVerlag.

De Villiers, M. (1997). The role of proof in investigative, computer-based geometry: Some personal reflections. In J. King \& D. Schattschneider, Geometry turned on: Dynamic software in learning, teaching, and research (pp. 15-24). Washington, DC: Mathematical Association of America.

De Villiers, M. (2003). Rethinking proof with Sketchpad. Emeryville: Key Curriculum Press.

Gilbert, G. T., Krusemeyer, M., \& Larson, L. C. (1993). The Wohascum County problem book. Dolciani Mathematical Expositions, 14(10), 68-70, Washington, DC: Mathematical Association of America.

Movshovitz-Hadar, N. (1988). Stimulating presentations of theorems followed by responsive proofs. For the Learning of Mathematics, 8(2), 12-19,30.

Pólya, G. (1945). How to solve it. A new aspect of mathematical method. Princeton, NJ: Princeton University Press.

National Council of Teachers of Mathematics. (1991). Professional standards for teaching mathematics. Reston, VA: National Council of Teachers of Mathematics.

Wales, J. (2010). Centroid. Retrieved from http://en.wikipedia.org/wiki/Centroid 